See discussions, stats, and author profiles for this publication at: https://www.researchgate.net/publication/336073827

\title{
System Identification in Resilience Management of Historical Bridges
}

Conference Paper · September 2019

DOI: 10.3850/978-981-11-2724-3_1070

CITATIONS

0

3 authors, including:

Zehra Irem Turksezer

Politecnico di Milano

7 PUBLICATIONS 9 CITATIONS

SEE PROFILE
READS

78

2.) Maria Pina Limongelli

88 PUBLICATIONS 416 CITATIONS

SEE PROFILE

Some of the authors of this publication are also working on these related projects:

COST Action TU1402: Quantifying the Value of Structural Health Monitoring View project

COST TU1406 Quality specifications for roadway bridges, standardization at a European level (BridgeSpec) View project 


\title{
Zehra Irem Turksezer
}

Department of Architecture, Built Environment and Construction Engineering, Politecnico di Milano, Italy. Email:zehrairem.turksezer@polimi.it

\section{Maria Pina Limongelli}

Department of Architecture, Built Environment and Construction Engineering, Politecnico di Milano, Italy. Email: mariagiuseppina.limongelli@polimi.it

\author{
Michael Havbro Faber \\ Department of Civil Engineering, Aalborg University,Denmark.E-mail: mfn@civil.aau.dk
}

Transportation infrastructures play an essential role for sustainable and resilient societal developments. In case of an infrastructure failure, such as a partial of full collapse of a bridge, the failure event may cause further cascading failures in the transportation network and lead to severe disruptions of societal functionality. The identification of optimal strategies for the management of critical infrastructure systems through inspections, monitoring, maintenance, repairs, strengthening and renewals, comprises a common but non-trivial challenge for asset owners and operators. To support assets integrity managers in this process the JCSS (2008) has issued a guideline on risk informed decision making for engineered facilities and systems with a focus on the main components of the system identification pertaining to decision analysis. With this basis, the present paper identifies and proposes a novel and generic framework for system identification in the context of resilience management of bridges with a special focus on heritage structures subject to disturbance events such as natural hazards, excessive degradation, failure and accidents and human and organizational errors. The framework addresses the decision situations before, during and after disturbance events with special emphasis directed on the system characteristics of relevance for resilience management and how information about these characteristics may be represented in terms of resilience indicators. In addition, the suggested framework directs emphasis on the role of management of information utilized in support of decision making.

Keywords: Resilience management, systems identification, historical bridges, resilience indicators, decision situations, information management.

\section{Introduction}

Resilience of socio-ecological-technical systems as a research area but also as a political focus for strategic and operational decision support for the management of critical societal infrastructure systems has gained significant interest during the last 1-2 decades (see e.g. Gardoni (2019) for a comprehensive overview).

In accordance with Faber (2019) and Faber et al. (2017) resilience of systems may be adequately modelled in the context of providing decision support as illustrated in Figure 1. A main issue here is that the modeling of systems resilience performance must account for the balance between the capacities of the systems and the disturbances which may exhaust these capacities. Moreover, the capacities of a systems must be related to the services provided by the systems.

In Faber et al. (2017) it is described how the framework illustrated in Figure 1 may be applied to support decisions on the life-cycle-based management of systems, accounting for safety, robustness, resilience, welfare and sustainability.

One of the major challenges in resilience modeling concerns the identification of the systems to be modelled, i.e. the systems identification. In the present paper we address this challenging and important issue in the context of heritage structures comprising part of roadway infrastructure systems. To this end we introduce the concept of indicators of resilience, along the lines of indicators of vulnerability and robustness addressed in JCSS (2008).

Section 2 first provides a short outline of the systems definition proposed by the JCSS (2008).

Proceedings of the 29th European Safety and Reliability Conference.

Edited by Michael Beer and Enrico Zio

Copyright (c) 2019 European Safety and Reliability Association.

Published by Research Publishing, Singapore.

ISBN: 978-981-11-2724-3; doi:10.3850/978-981-11-2724-3_1070-cd 


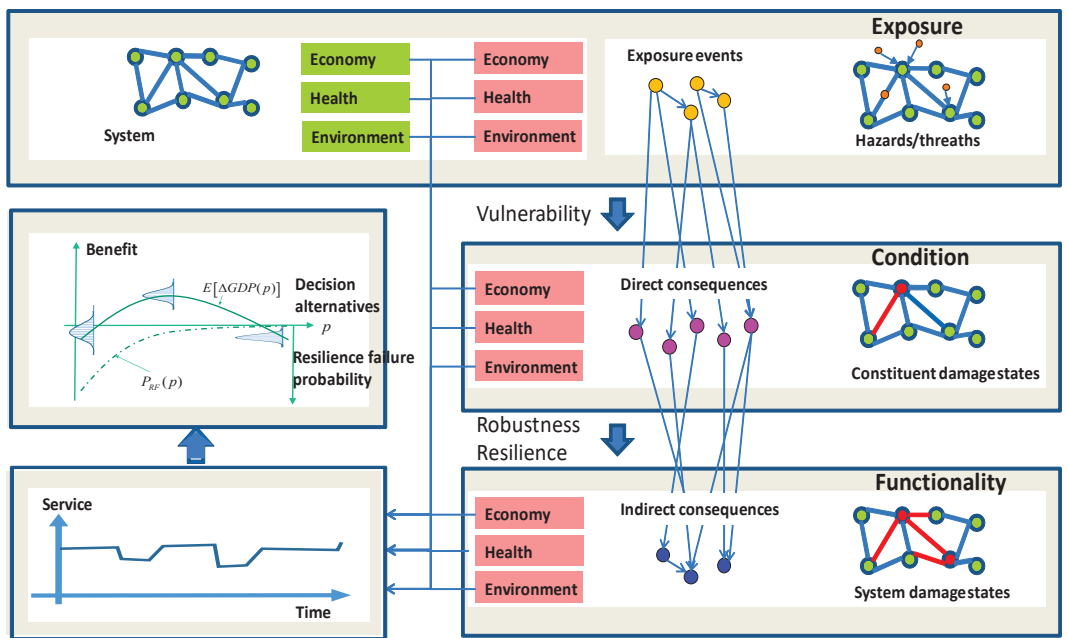

Fig.1 Framework for resilience modeling of socio-ecological-technical systems

Thereafter in Section 3, to underline the importance of the management of the organizational systems, a discussion of resilience management is provided taking basis in a selection of examples from past events of disturbances of infrastructure systems. In Section 4, a framework for categorization of systems resilience indicators is introduced as a means to facilitate resilience modeling based on observable system characteristics. In Section 5 the proposed framework is applied for the case of historical roadway bridges and finally in Section 6 conclusions and an outlook regarding further research are provided.

\section{Definition of the System}

A system can comprise several constituents such as assets (e.g. structures, infrastructures), their constituents (e.g. technical equipment), humans, environment and operational process (JCSS 2008) and provides certain functionalities (e.g. physical, social, economic, cultural, environmental and organizational).

In the framework for resilience management proposed in this paper the constituents of the system are grouped in three subsystems namely physical, information and organization.

The physical sub-system is managed by decision-makers who are part of the organizational sub-system (see Figure 2). Decisions on the management of the physical sub-system are enabled and supported by the information sub-system. In accordance with JCSS (2008) the life-time of the physical subsystem may be considered as a sequence of before, during and after decision situations centered around the occurrences of disturbance events.

\subsection{Decisions making}

The decision maker aims to attain some objectives that are in balance with the resources allocated by the decision. Each decision made by decision maker between several decision alternatives, by weighing different attributes, may generate different consequences in terms of potential benefits and losses. The objectives represent preferences of the decision maker. Thus, each decision relies on objectives, preferences and attributes. The attributes to be used should describe what is indicated by the objective. The objectives must be quantified with utility model after choosing the set of attributes. It is possible by transforming the attribute values to a non-dimensional scale which is often referred as a utility function (JCSS 2008).

\subsection{Constituents of the system}

Physical system includes all components that can affect structural reliability: structural constituents, non-structural constituents and environment, that may originate exposure and can be influenced by the direct and indirect consequences. The physical system can refer to a single structure, a group of structures or a network.

Information system purposes to get and transmit true information (Nielsen et al. 2018) in the intended amount, format and time to the relevant people. It includes technological 
constituents (computer-based tools and techniques to collect, store and process data and information) and non-technological constituents (legacy data that is difficult to process due to its old or obsolete format). Legacy data describe the history of the structure (past interventions, past hazard records, and the activities of previous operators or owners).

The information system also includes (Rainer and Cegielski 2009),

- data, which is the observation and measure of the system, recorded and classified by symbols, numbers, images or figures;

- information is the data processed to describe the state of the system;

- knowledge consists of information which are processed and transformed by human being to experience or expertise.

Organization system has a hierarchical structure that includes governance, managing the services of the three sub-systems, and comprises several levels.

The hierarchical structure consists of several stakeholders which are decision makers and third parties who are affected by decisions. Decision makers are at global, national and local levels. At the first two levels (e.g. politicians, regulatory bodies, civil protection) they are responsible of managing the entire system and creating engineering codes and standards. At local level e.g. municipalities or regions, private or public asset owners, managers or operators, bodies and agencies in charge of emergency management (e.g. first responders, civil servants), professionals (e.g. engineers, architects and urban planners), public/private media, users.

The preferences and freedom of the decision maker comprise and rely on the perception of stakeholders (Nielsen et al. 2018). This is why there are crucial dependencies among stakeholder, decision makers and information in the decision making.

\subsection{Operation of the system}

Data, information and knowledge flow between the three sub-systems supporting decision making and management activities. Data relevant to the physical system is collected, processed, transmitted and transformed to information by the technological and non-technological constituents of the information system (e.g. coordination, communication and emergency plans). The legacy data may or may not be used after translation into technological constituents.

Information is transmitted to the organizational sub-system and, at each level inside the organization, is gradually turned into knowledge by the human factor (people on operational levels, users, third parties) connected with decision making. Information is also translated into knowledge between stakeholders by using networks (e.g. coordination, communication and emergency plans).

The information that cannot be processed at a certain level is transmitted unchanged to the higher levels to be utilized for decision making. Therefore, when the flow arrives to governance level, there are large amounts of knowledge with a little amount of unprocessed information.

Knowledge can be employed immediately as decision support or can be kept by the population as manufactured culture - as basis for directing future decisions. In this way, long-term learning can be provided by memories from previous events which keep the knowledge and experiences alive over generations (Giuliani et al. 2016).

Between the stakeholders, private and public media have a significant position to transfer information and knowledge between stakeholders and thus, they help to bring the state closer to people during emergencies (Boin and Connell 2007). As stated before, several hazards resulted in catastrophes due to miscommunication between stakeholders. By using information and knowledge, organizational systems can develop and improve emergency and communication plans which may contribute to a resilient system.

\subsection{Disruptive events}

During the service life, every system performs under certain risks and undergoes one or more sudden or gradual hazardous events (exposures) and eventually experience service disruptions, that is a disruptive event.

The disruptive event can be modelled using the JCSS framework that identifies the event scenario that is composed by the sequence of: a) the exposure event that starts from the moment of exposure and includes all actions on the constituents of the system; b) direct consequences associated with damages and 
failures of the system constituents; c) indirect consequences related to the functionality loss of the system.

Disruptive event can originate from sudden exposures, such as natural events, contingencies or manmade mishaps or from slowly-varying actions due to climatic changes, environmental effects (e.g. corrosion) or deterioration due to aging. Consequences vary depending on the characteristic (magnitude, duration, etc.) of the exposure, the location where the disruptive event occurs, and the assets exposed.

Risk-based decision making (JCSS 2008) aims to assess and manage risks in three event phases. Before phase is the time between the last intervention to re-establish functionality at the required level and the time the disruptive event occurs. The during phase is the time between the start of the disruption and the start of the recovery. The after phase is the time between the start and the end of the recovery.

In the before phase, the concern of the decision maker is to optimize investments into preventive measures. During an event, the aim of the decision maker is to limit the consequences; while after a hazardous event the issue is to rehabilitate the losses, to adapt and recover the functionalities and to reevaluate what have been done to reorganize the system.

The cycle of these three phases may repeat several times during the service life of the system. Thus, it can be said that the 'after' phase of a cycle coincides with the 'before' phase of the next one.

\section{Resilience Management}

According to Faber (2019) the objective of societal decision makers and stakeholders at each level of the societal governance hierarchy is to provide and document adequate resilience of the systems under their responsibility. The significance and challenges associated with this objective are demonstrated through several disruptive events over the last few decades that led to resilience failures which may be assigned to mismanagement of the organizational system.

For example, in 2005, Hurricane Katrina started with a hurricane and transformed to a big catastrophe due to the failure in communication between leaders, local responsible and federal organizations. The exposure lasted longer due to the consecutive disruptions in the system.
Another hurricane was Rita, in the same year which created massive traffic congestions during the unplanned evacuation after the hurricane and finalized with suffering people on roads without fuel, food or any information (Litman 2006).

The importance of awareness and preparedness of governance has been seen in 2009 L'Aquila earthquake. It resulted in organizational failure and extensive losses that were not recovered even after many years, i.e. in long-term recovery (Alexander 2010).

For heritage structures, functionalities may range from technical, to economic ones linked to touristic activities, up to intangible functionalities related to the cultural and social value of these structures. For instance, during the war in Bosnia in 1993, the Mostar bridge which had been built in the $16^{\text {th }}$ century was destroyed by a three days-long bombing. This bridge had been seen as the symbol of multi-ethnicity of the city besides providing connection to pass Neretva River and it was destroyed to demolish its symbolic significance. After the destruction of Mostar bridge, for 11 years a temporary bridge was used and in 2004, it was decided to reconstruct a replica of the old bridge to reunite the community, increase the tourism related activities in the area and keep the war related memories of the people alive (Armaly et al. 2004). Although there was no organizational failure and a temporary bridge was used for a long time, the cultural service provided by the historical bridge was lost.

Another example to cultural loss is Santa Trinita bridge in Florence which was destroyed by bombing during the $2^{\text {nd }}$ World War. This bridge was originally rebuilt after the war with the materials saved (Armaly et al. 2004). Even though the structure was reconstructed as a symbol of history and remembrance, its previous cultural value was lost with the destroyed bridge.

\section{Indicators}

Indicators can be considered as the instruments transmitting specific information on the state of the constituents of the system to the resilience management. The state of all the constituents (i.e. physical, informative and organizational) influences the resilience of the system. For this reason, resilience indicators are set considering each constituent in the different phases, see Table 1. Although governance has been defined 
in the foregoing as the inclusive constituent of the system, it is included in the table individually for the three different phases to highlight the difference of the corresponding relevant indicators.

Six main indicators have been selected to describe the performances of the system constituents in the three-time phases i.e.: resistance, ductility, diversity, redundancy, robustness and efficiency. For each indicator a common definition across the constituents and the time phases has been proposed (see Table 1).

Direct consequences depend on the resistance of the system whereas indirect consequences are related to the system robustness. The latter on its turn depends on the system ductility, redundancy and diversity.

Resistance is the ability to meet demand without irreversible changes to constituents. Robustness is the ability to limit the indirect consequences of damage and failures of constituents. Ductility is the ability to meet demand after reaching the level where the system sustains irreversible changes to constituents. Redundancy is the ability to meet the same demand by different constituents. Diversity is the ability to meet different demands by one or various constituents.

As mentioned in the previous section the ability of the system to provide functionality with minimum investment is crucial for the systems resilience: this ability is herein represented by the indicator efficiency. In order to describe the means that provide these abilities to the three constituents in the three different phases, several sub-indicators have been proposed for each indicator. For example, redundancy can be provided by alternative routes or other structures in the network able to provide service the same level of transportation or available physical and financial resources at the system level to keep management running. Diversity at the network level is represented by several available routes connecting two points of the network. At the system level alternative decisions provide diversity to the decision makers.

\section{Framework for historical bridges}

In the following sections, the general framework for transportation networks presented in the previous sections will be applied for the case of historic bridges.

There are two key features that distinguish historical from ordinary bridges: they have further functionalities beside transportation and the spectrum of possible alternative decisions is limited. Historical bridges provide cultural (knowledge about traditions, art, construction techniques), social (understanding and storing the memories of people supporting lifelong learning), economic services (tourism related activities) and environmental (reuse) services. These services are interrupted by the partial or total collapse of the bridge and not recoverable by reconstruction due to their uniqueness. Secondly, for historical bridges the spectrum of decisions on maintenance, restoration, reconstruction or demolishment is bounded by requirements to consider minimum interventions and non-destructive techniques. These are required by regulations and standards at national and global levels, due to the cultural value of the structure. In this case, the physical system is a historical bridge and the non-structural constituents are the ones affecting reliability and the environment.

The information system, beside the constituents listed in section 2.3 includes the manufactured culture constituted by the architectural and engineering techniques as well as materials embedded in the structure itself.

The organizational system includes operational levels starting from lower levels namely project management which is in charge of individual projects (e.g. single bridges or infrastructure systems, communication technologies, local emergency management etc.); overlying levels as network management which are responsible of portfolio management of several projects. The sector management level has the authority for making decisions on larger scale projects that include several networks. The overall governance is considered above all these levels on the national or global level, as shown in Figure 2. Decision makers at different levels of the organizational system operate at different ranges of time, space and knowledge.

Before any disruptive event occurs, the project manager of a historical bridge is responsible of maintenance, planning and budgeting for a specific time period, for instance for 5 years. During and after a disruptive event, 
the project management level can only make decisions to limit the direct consequences to the individual project in their domain. Network managers plan for longer time ranges (e.g. lifecycle of the transportation network) for the entire transportation network and in case of a disruptive event, aim to limit the indirect consequences on the system. Besides, sector management (or governance level) is expected to decide by looking at the overall picture in longer time period (e.g. lifecycle of the whole system) and in larger domain such as in local or national level. Disruptive events originating from external demands (e.g. transportation, wind, seismic, etc.) or degradation phenomena may lead to the partial or total collapse of the historical bridge.
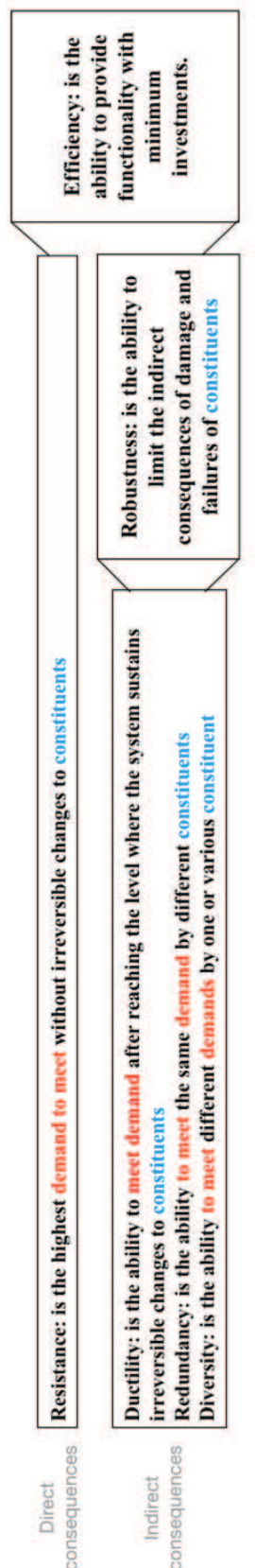
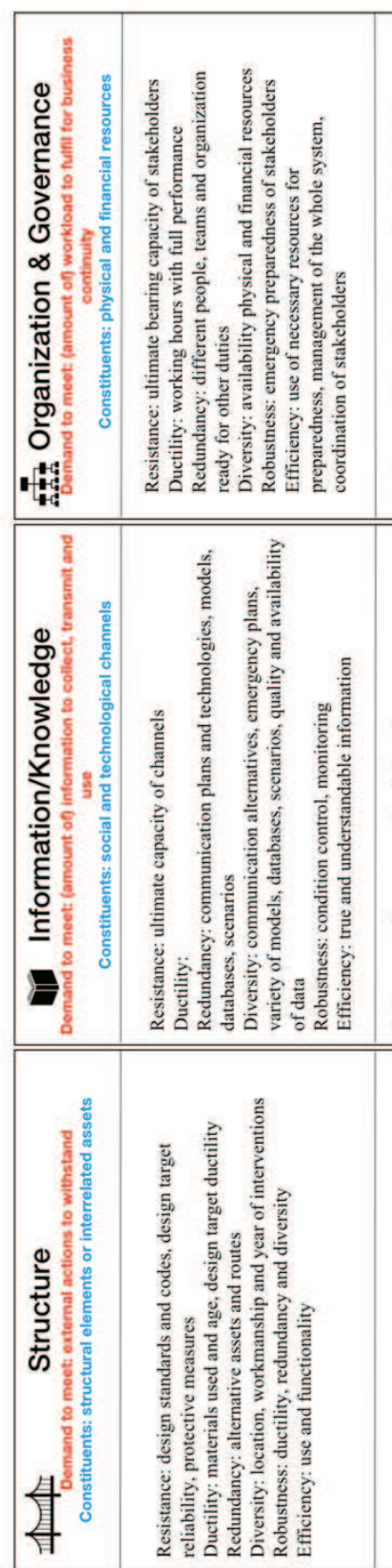

벙코
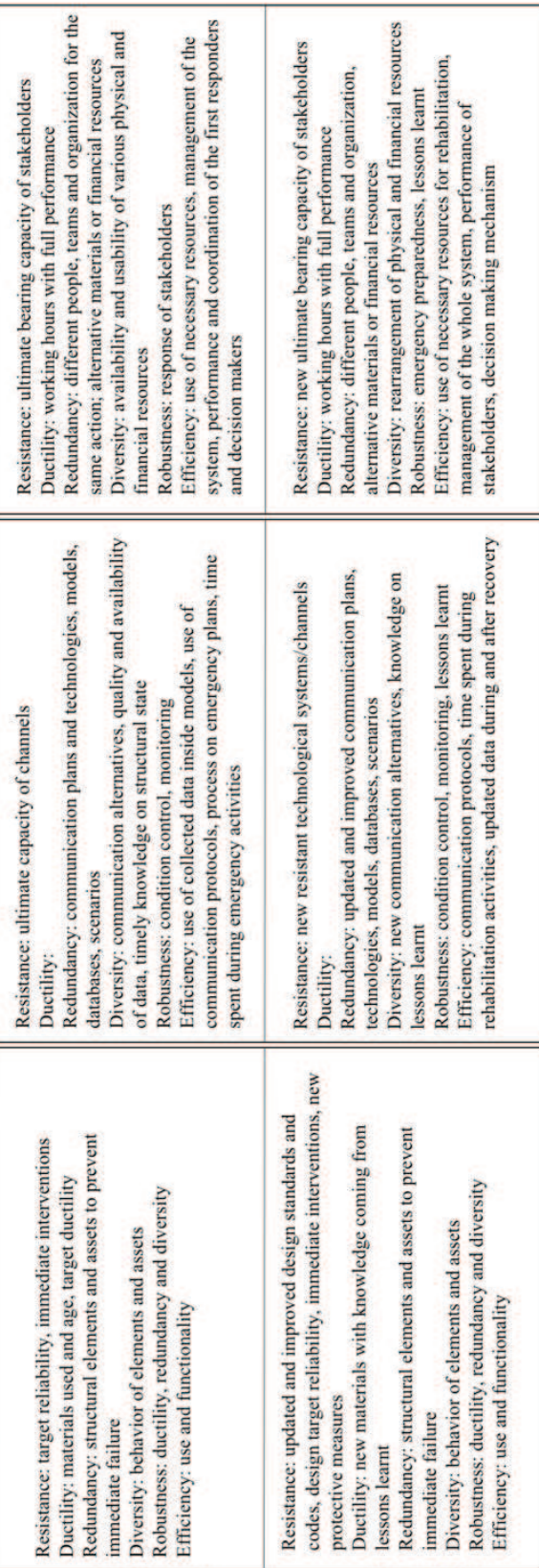

כNIYกa

प्木

Table 1. Resilience Indicators 
With reference to the three time phases defined in section 2.4 (before, during and after) the duration of the during phase, for example the collapse of the bridge, can be defined as: a) the time between the start of the traffic disruption and the formalization of a different route; b) the time between the start and the end of the damaging phenomena (the end could coincide with the arrival of the rescue teams). Here in all cases, the type of the disruption and its consequences are the same, however the time interval varies from days to hours.

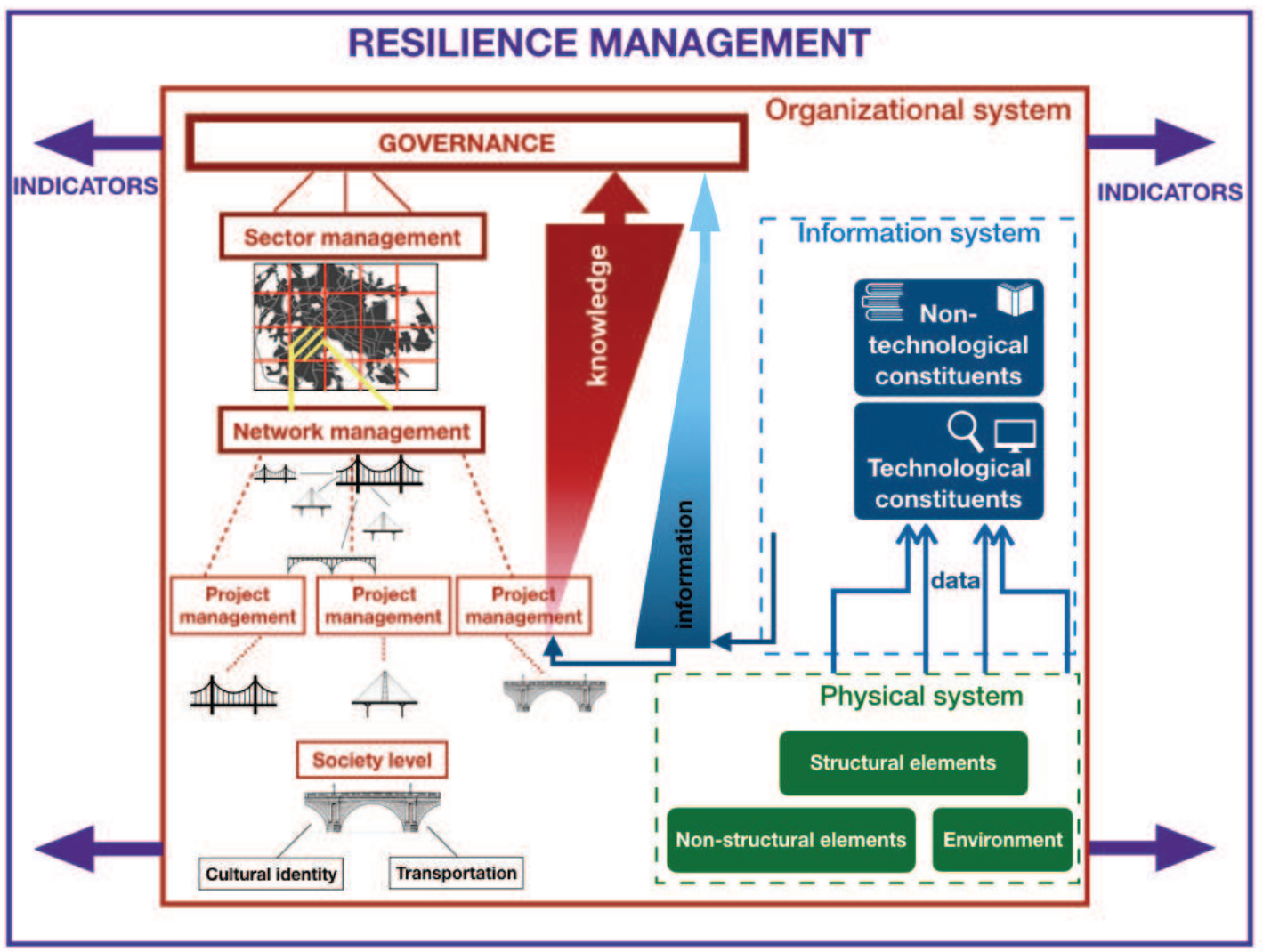

Fig. 2. System representation for historical bridges

When partial or total collapse of historical bridge is considered, the direct consequences on the physical system are the structural and material losses.

The indirect consequences are 1) partial or total functional loss in transportation network system with consequences in terms of time and cost; 2) physical damage on the surrounding assets (e.g. buildings, roadway or railway passing under the bridge, other critical infrastructures (CIs) like electricity, pipelines etc.); 3) damage to environment in local level as debris, pollutants, animal deaths and in global level increase in fuel consumption and gas emissions due to vehicles waiting on the blocked roads or using longer routes to avoid blockage; 4) loss of lives and injuries; 5) losing possibility to reach information about the structure itself.
For the information system the direct consequences are 1) damage on technological constituents placed on the bridge; 2) loss of knowledge about the bridge - manufactured culture - including information or knowledge on inadequate maintenance, negligence of utility operator, un-robust structural elements etc. In case of lack of written records (or archive documents) about the historical bridge, full collapse precludes data collection on the structural behavior; material characteristics, structural geometry to provide structural plans; and structural constituents.

The indirect consequences on the information system involve 1) no data transfer from bridge to informative system, therefore disruption or delay on other technological constituents e.g. databases, models, scenarios etc. and thus 2) 
communication loss between organizational levels.

In the organizational system the direct consequences are: 1) disorganization between stakeholders due to communication disruption; 2) loss of trust toward authorities; 3) business interruption and monetary loss due to activities of interdependent CIs such as roadway, port etc.; 4) loss in terms of users' time and money since they are obliged to shunt, thus increasing risks due to ascending traffic on alternative routes; 5) fear and despair in the population which may lead to psychological damages. Indirect consequences can be considered at the governance level such as guilt, need to change and update design standards and codes as well as to reorganize lower organizational levels and update their responsibilities.

\section{Conclusion}

In this paper a novel framework to model the resilience management of bridges is presented. Three constituents of the system namely physical, information and organization are addressed in three event phases i.e. before, during and after disruptive events.

Operations and resourcefulness of the system is provided by the flow of data, information and knowledge among the system components. Indicators are defined as tools to feed resilience management by transmitting information about the abilities of the system constituents relevant to the resilient behavior of the system.

This framework is new, and several aspects have to be investigated in more detail. However, it is general and could be applied to diverse types of systems. Herein it has be applied to the case of historical bridges that present the specificities related their historical meaning and cultural functionality. These introduces a different perspective in the resilience management that should account for the irreplaceable interruption of function in case of the collapse of the bridge.

\section{Acknowledgement}

The networking support of Cost Action 1406Quality Specifications for Roadway Bridges, Standardization at a European Level, is gratefully acknowledged.

\section{References}

Alexander, D. (2010). The L'Aquila earthquake of 6 April 2009 and Italian Government policy on disaster response. Journal of Natural Resources Policy Research 2 (4): 325-342.

Armaly, M., C. Blasi, and L. Hannah (2004). Stari Most: rebuilding more than a historic bridge in Mostar. Museum International 56 (4): 6-17.

Boin, A., and A. McConnell. "Preparing for critical infrastructure breakdowns: the limits of crisis management and the need for resilience." Journal of Contingencies and Crisis Management 15, no. 1 (2007): 50-59.

Faber, M. H. (2019). On sustainability and resilience of engineered systems. In Routledge Handbook of Sustainable and Resilient Infrastructure, edited by Paolo Gardoni, 28-49. New York: Routledge.

Faber, M. H., J. Qin, S. Miraglia, and S. Thöns (2017). On the probabilistic characterization of robustness and resilience. Procedia engineering 198: 10701083.

Gardoni, P. (Ed.) (2019) Routledge Handbook of Sustainable and Resilient Infrastructure, edited by Paolo Gardoni. New York: Routledge.

Giuliani, L., A. Revez, J. Sparf, S. Jayasena, and M. H. Faber (2016). Social and technological aspects of disaster resilience. International Journal of Strategic Property Management 20 (3): 277-290

JCSS (2008). Risk assessment in engineering: principles, system representation \& risk criteria. ETH Zurich.

Nielsen, L., S.T. Glavind, J. Qin, and M. H. Faber (2018). Faith and Fakes - Dealing with critical information in decision analysis. Lake Louise: IFED Forum.

Rainer, K. and C. G. Cegielski (2009). Introduction to Information Systems: Enabling and Transforming Business. John Wiley \& Sons. 